\title{
RANDOM FIXED POINT THEOREMS FOR NONEXPANSIVE AND CONTRACTIVE-TYPE RANDOM OPERATORS ON BANACH SPACES ${ }^{1}$
}

\author{
ISMAT BEG \\ Kuwait University, Department of Mathematics, Kuwait \\ and Quaid-i-Azam University, Department of Mathematics, Islamabad, Pakistan \\ NASEER SHAHZAD ${ }^{2}$ \\ Quaid-i-Azam University, Department of Mathematics \\ Islamabad, Pakistan
}

(Received February, 1994; revised November, 1994)

\begin{abstract}
The existence of random fixed points for nonexpansive and pseudocontractive random multivalued operators defined on unbounded subsets of a Banach space is proved. A random coincidence point theorem for a pair of compatible random multivalued operators is established.
\end{abstract}

Key words: Random Fixed Point, Random Coincidence Point, Random Multivalued Operator.

AMS (MOS) subject classifications: $47 \mathrm{H} 10,47 \mathrm{H} 04,60 \mathrm{H} 25$.

\section{Introduction}

Random fixed point theorems for contraction mappings in Polish spaces were proved by Spacek [20], Hans [7, 8] and many others. For complete survey, we refer to Bharucha-Reid [3]. Itoh $[10,11]$ established several random fixed point theorems for various commuting single and multivalued random operators. Afterwards, Sehgal and Singh [18], Papageorgiou [17] and Lin [15] proved different stochastic versions of well-known approximation result of Fan [4] and obtained some random fixed point theorems. Recently, Beg and Shahzad [2] studied the structure of common random fixed points and random coincidence points of a pair of compatible random multivalued operators in Polish spaces. The purpose of this paper is to prove some random fixed point theorems for random multivalued nonexpansive and pseudocontractive operators defined on closed convex unbounded subsets of a Banach space. Section 2, is aimed at clarifying the terminology to be used and recalling some necessary definitions. In Section 3, the existence of random fixed points for nonexpansive random multivalued operators defined on an unbounded closed convex subset of a Banach space is established. A random fixed point theorem for Lipschitzian pseudocontractive operators is also proved. Section 4 contains a random fixed point theorem for a $\lambda(\omega)$-firmly nonexpansive random operator in separable Banach spaces. Section 5 deals with random coincidence point theorems for a pair of compatible random multivalued operators satis-

\footnotetext{
${ }^{1}$ Research supported by the NSRDB Research Grant No. M. Sc. (5)/QAU/90.
}

${ }^{2}$ Florida Institute of Technology, Program of Applied Mathematics, Melbourne, Florida 32901-6988, USA.

Printed in the U.S.A. (C)1994 by North Atlantic Science Publishing Company 
fying a contractive type condition.

\section{Preliminaries}

Throughout this paper, $(\Omega, \mathcal{A})$ denotes a measurable space. Let $(X, d)$ be a metric space, $\mathscr{P}(X)$ the family of all subsets of $X, \mathscr{K}(X)$ the family of all nonempty compact subsets of $X$, $\mathcal{C} \mathfrak{B}(X)$. the family of all nonempty closed bounded subsets of $X$ and $\mathcal{C} \mathfrak{B C}(X)$ the family of all nonempty closed bounded convex subsets of $X$. A mapping $T: \Omega \rightarrow \mathscr{P}(X)$ is called measurable if for any open subset $C$ of $X, T^{-1}(C)=\{\omega \in \Omega: T(\omega) \cap C \neq \phi\} \in \mathcal{A}$. A mapping $\xi: \Omega \rightarrow X$ is said to be a measurable selector of a measurable mapping $T: \Omega \rightarrow \mathscr{P}(X)$ if $\xi$ is measurable and for any $\omega \in \Omega, \xi(\omega) \in T(\omega)$. Let $M$ be a subset of $X$. A mapping $f: \Omega \times M \rightarrow X$ is called a random operator if for any $x \in M, f(\cdot, x)$ is measurable. A mapping $T: \Omega \times M \rightarrow \mathcal{C} \mathfrak{B}(X)$ is a random multivalued operator if for any $x \in M, T(\cdot, x)$ is measurable. A measurable mapping $\xi: \Omega \rightarrow M$ is called a random fixed point of a random multivalued (single-valued) operator $T: \Omega \times M \rightarrow \mathrm{CFB}(X)$ $(f: \Omega \times M \rightarrow X)$ if for every $\omega \in \Omega, \xi(\omega) \in T(\omega, \xi(\omega))(\xi(\omega)=f(\omega, \xi(\omega)))$. A measurable mapping $\xi: \Omega \rightarrow M$ is a random coincidence point of $T: \Omega \times M \rightarrow \mathcal{C B B}(X)$ and $f: \Omega \times M \rightarrow X$ if for every $\omega \in \Omega$, $f(\omega, \xi(\omega)) \in T(\omega, \xi(\omega))$. A mapping $T: M \rightarrow \mathfrak{C} \mathfrak{B}(X)$ is upper (lower) semicontinuous if for any closed (open) subset $C$ of $M, T^{-1}(C)$ is closed (open). A mapping $T$ is called continuous if $T$ is both upper and lower semicontinuous. A mapping $T: M \rightarrow \mathrm{CF}(X)$ is called Lipschitzian if $H(T x, T y) \leq k d(x, y)$ for any $x, y \in M, k \geq 0$, where $H$ is the Hausdorff metric on $\mathfrak{C F}_{\mathfrak{B}}(X)$, induced by the metric $d$. When $k<1(k=1)$ then $T$ is called contractive (nonexpansive). A mapping $T: M \rightarrow \mathfrak{C B B}(X)$ is pseudocontractive if for any $x, y \in M, u \in T x, v \in T y, r>0$, we have

$$
\|x-y\| \leq\|(1+r)(x-y)-r(u-v)\| .
$$

A random operator $T: \Omega \times M \rightarrow \mathfrak{C B B}(X)$ is pseudocontractive if $T(\omega, \cdot)$ is pseudocontractive for each $\omega \in \Omega$ (in this case, $r: \Omega \rightarrow[0, \infty)$ is a measurable mapping). Following Itoh [10], a measurable mapping $L: \Omega \rightarrow[0, \infty)$ is called Lipschitz measurable mapping of $T$ if, $H(T(\omega, x), T(\omega, y)) \leq$ $L(\omega) d(x, y)$. Let $T: \Omega \times M \rightarrow \mathcal{C} \mathfrak{B}(X)$ be a random operator and $\left\{\xi_{n}\right\}$ be a sequence of measurable mappings $\xi_{n}: \Omega \rightarrow M$. The sequence $\left\{\xi_{n}\right\}$ is said to be asymptotically T-regular if $d\left(\xi_{n}(\omega)\right.$, $\left.T\left(\omega, \xi_{n}(\omega)\right)\right) \rightarrow 0$ for each $\omega \in \Omega$. Mapping $T: M \rightarrow \mathfrak{C B B}(X)$ is said to be demiclosed if the conditions that $x_{n}$ converges weakly to $x, y_{n}$ converges to $y$, and $y_{n} \in T x_{n}$, imply that $y \in T x$. For any $A \in \mathfrak{C} \mathfrak{B}(X)$, we denote with $\overline{C o}(A)$ the closed convex hull of $A$.

\section{Random Fixed Points for Random Multivalued Operators Defined on Unbound- ed Sets}

In 1978, Goebel and Kuczumov [5] proved that, if $X$ is a closed convex subset of $\ell_{2}$ and $T: X \rightarrow X$ is nonexpansive for which there exists a point $x \in X$ such that the set

$$
L S(x, T x ; X):=\{z \in X:\langle z-x, T x-x\rangle \geq 0\}
$$

is bounded, then $T$ has a fixed point in $X$. Kirk and Ray [13] have shown that if $X$ is an unbounded closed convex subset of a uniformly convex space and $T: X \rightarrow X$ is a Lipschitzian pseudocontractive mapping for which the set

$$
G(x, T x ; x):=\{z \in X:\|z-T x\| \leq\|z-x\|\}
$$

is bounded for some $x \in X$, then $T$ has a fixed point in $X$. Subsequently, Marino [16] extended 
these results to the multivalued case and improved some known results.

Let $X$ be a real Banach space and let $K$ be a nonempty convex subset of $X$. We set (for any $x, y \in X)$

$$
\tau(x, y):=\lim _{t \rightarrow 0^{+}} \frac{\|x+t y\|-\|x\|}{t} .
$$

Following [16] we define, for $x, y \in X, \epsilon>0, A \subseteq X$,

$$
\begin{gathered}
L S(x, y ; K):=\{z \in K: \tau(x-z, y-x)<0\} \\
L S(x, y, \epsilon ; K):=\{z \in K: \tau(x-z, y-x)<\epsilon\} \\
L S(x, A ; K):=\{z \in K: \exists a \in A: \tau(x-z, a-x)<0\} \\
=\bigcup_{\alpha \in A} L S(x, a ; K)
\end{gathered}
$$

and

$$
\begin{aligned}
L S(x, A, \epsilon ; K):= & \{z \in K: \exists a \in A: \tau(x-z, a-x)<\epsilon\} \\
& =\bigcup_{\alpha \in A} L S(x, a, \epsilon ; K) .
\end{aligned}
$$

The aim of this section is to establish random fixed point theorems for nonexpansive and pseudocontractive random operators defined on unbounded sets in Banach spaces.

Theorem 3.1: Let $X$ be a separable closed convex subset of a real Banach space, and let $T: \Omega \times X \rightarrow \mathscr{H}(X)$ be a nonexpansive random operator. Suppose that for some bounded set $W \subseteq X$ the set $L S(W, T(\omega, W) ; X):=\bigcap_{z \in W} L S(z, T(\omega, z) ; X)$ is bounded for each $\omega \in \Omega$. Then there exists a bounded sequence $\left\{\xi_{n}\right\}$ of measurable mappings $\xi_{n}: \Omega \rightarrow X$ which is asymptotically T-regular.

Proof: Choose an element $y \in X$ and a sequence $\left\{\alpha_{n}\right\}$ of measurable mappings $\alpha_{n}: \Omega \rightarrow(0,1)$ such that $\alpha_{n}(\omega) \rightarrow 1$ as $n \rightarrow \infty$ pointwise in $\omega$. For each $n$, define a contractive random operator $T_{n}: \Omega \times X \rightarrow \mathscr{K}(X)$ by $T_{n}(\omega, x):=\left(1-\alpha_{n}(\omega)\right) y+\alpha_{n}(\omega) T(\omega, x)$. Then by Itoh [10], $T_{n}$ has a random fixed point $\xi_{n}$. Assume that the set $\left\{\xi_{n}(\omega)\right\}_{n \in N}$ is unbounded. Then it is possible to choose $k \in N$ such that for each $\omega \in \Omega$,

$$
\sup _{z \in W} H(\{y\}, T(\omega, z))<d\left(\xi_{k}(\omega), W\right)
$$

and

$$
\sup _{p \in L S(W, T(\omega, W) ; X)}\|p\|<\left\|\xi_{k}(\omega)\right\| .
$$

We will prove that for any $z \in W$ and $\omega \in \Omega$, there exists $x_{z} \in T(\omega, z)$ such that $\tau\left(z-\xi_{k}(\omega), x_{z}\right.$ $-z)<0$. Indeed, by $\xi_{k}(\omega) \in\left(1-\alpha_{k}(\omega)\right) y+\alpha_{k}(\omega) T\left(\omega, \xi_{k}(\omega)\right)$, it follows that

$$
\xi_{k}(\omega)=\left(1-\alpha_{k}(\omega)\right) y+\alpha_{k}(\omega) \eta_{k}(\omega), \quad \eta_{k}(\omega) \in T\left(\omega, \xi_{k}(\omega)\right)
$$

for each $\omega \in \Omega$. (The existence of measurable maps $\eta_{k}: \Omega \rightarrow X$ is due to Kuratowski and Ryll-Nardzewski [14].) From the nonexpansivity of $T$, there exists $x_{z} \in T(\omega, z)$ such that for any $\omega \in \Omega$, $\left\|\eta_{k}(\omega)-x_{z}\right\| \leq\left\|\xi_{k}(\omega)-z\right\|$ and therefore $\tau\left(z-\xi_{k}(\omega), x_{z}-z\right)<0$ for each $\omega \in \Omega$ [16, see proof of Theorem 2]; that is, $\xi_{k}(\omega) \in L S(W, T(\omega, W) ; X)$, contradicting (2). Thus, $M(\omega)$ : = $\sup \left\{\left\|\xi_{n}(\omega)-y\right\|: n \in N\right\}<\infty$ for all $\omega \in \Omega$, (the mapping $M: \Omega \rightarrow \mathbb{R}^{+}$is measurable) and 
moreover,

$$
d\left(\xi_{n}(\omega), T\left(\omega, \xi_{n}(\omega)\right)\right) \leq\left(\frac{1-\alpha_{n}(\omega)}{\alpha_{n}(\omega)}\right)\left\|\xi_{n}(\omega)-y\right\| \leq\left(\frac{1-\alpha_{n}(\omega)}{\alpha_{n}(\omega)}\right) M(\omega) \rightarrow 0 \text { as } n \rightarrow \infty .
$$

Theorem 3.2: Let $X$ and $T$ be as in Theorem 3.1. Suppose that, for some bounded set $W \subseteq X$ and $\epsilon>0$, the set

$$
L S(W, T(\omega, W), \epsilon ; X):=\bigcap_{z \in W} L S(z, T(\omega, z), \epsilon ; X)
$$

is relatively compact for each $\omega \in \Omega$. Then $T$ has a random fixed point.

Proof: Let $y \in X$ and $A(\omega):=\sup \{\|y-x\|: x \in T(\omega, W)\}$. Then, for each sequence $\left\{\alpha_{n}\right\}$ of measurable mappings $\alpha_{n}: \Omega \rightarrow(0,1)$ such that for any $\omega \in \Omega, \alpha_{n}(\omega) \in I(\omega):=\left(\frac{A(\omega)-\epsilon}{A(\omega)}, 1\right) \cap(0,1)$ and $\alpha_{n}(\omega) \rightarrow 0$ as $n \rightarrow \infty$, we define a contractive random operator $T_{n}: \Omega \times X \rightarrow \mathscr{K}(X)$ by $T_{n}(\omega, x):=\left(1-\alpha_{n}(\omega)\right) y+\alpha_{n}(\omega) T(\omega, x)$. As in Theorem 3.1, $T_{n}$ has a random fixed point $\xi_{n}$, that is, $\xi_{n}(\omega) \in T\left(\omega, \xi_{n}(\omega)\right)$ for each $\omega \in \Omega$. For any $z \in W$, there exists $x_{z} \in T(\omega, z)$, such that

$$
\left\|\xi_{n}(\omega)-x_{z}\right\| \leq H\left(T_{n}\left(\omega, \xi_{n}(\omega)\right), T(\omega, z)\right)
$$

for $\omega \in \Omega$. Therefore, $\tau\left(z-\xi_{n}(\omega), x_{z}-z\right)<\epsilon\left[16\right.$, see proof of Corollary 3]. Hence, $\xi_{n}(\omega) \in$ $L S(W, T(\omega, W), \epsilon ; X)$ for each $\omega \in \Omega$. By Theorem 3.1, we have $d\left(\xi_{n}(\omega), T\left(\omega, \xi_{n}(\omega)\right)\right) \rightarrow 0$ as $n \rightarrow \infty$. For each $n$, define $F_{n}: \Omega \rightarrow \mathscr{G}(X)$ by $F_{n}(\omega)=c \ell\left\{\xi_{i}(\omega): i \geq n\right\}$, where $c \ell(C)$ is the closure of $C$. Let $F: \Omega \rightarrow \mathscr{K}(X)$ be a mapping defined by $F(\omega)=\bigcap_{n} F_{n}(\omega)$. The mapping $F$ is measurable by Himmelberg [9, Theorem 4.1]. Thus by Kuratowski and Ryll-Nardzewski [14] there is a measurable selector $\xi^{*}$ of $F$. Fix $\omega \in \Omega$ arbitrarily, then some subsequence $\left\{\xi_{k}(\omega)\right\}$ of $\left\{\xi_{n}(\omega)\right\}$ converges to $\xi^{*}(\omega)$. Thus $\xi^{*}$ is a random fixed point of $T$.

Corollary 3.3: Let $X$ be a separable closed convex subset of a reflexive (real) Banach space, $T: \Omega \times X \rightarrow \mathscr{H}(X)$ be a nonexpansive random operator, and for each $\omega \in \Omega, I-T(\omega, \cdot)$ be demiclosed on $X$. Suppose that, for some bounded set $W \subseteq X$ the set $L S(W, T(\omega, W) ; X)$ is bounded for each $\omega \in \Omega$. Then, $T$ has a random fixed point.

Proof: As in the proof of Theorem 3.1, there exists a bounded sequence of measurable mappings $\xi_{n}: \Omega \rightarrow X$ such that

$$
\xi_{n}(\omega)=\left(1-\alpha_{n}(\omega)\right) y+\alpha_{n}(\omega) \eta_{n}(\omega) \text { with } \eta_{n}(\omega) \in T\left(\omega, \xi_{n}(\omega)\right)
$$

for each $\omega \in \Omega,\left\{\eta_{n}(\omega)\right\}$ is also bounded, and $\left\|\xi_{n}(\omega)-\eta_{n}(\omega)\right\|=\left(1-\alpha_{n}(\omega)\right)\left\|\eta_{n}(\omega)\right\| \rightarrow 0$ as $n \rightarrow \infty$. Fix $\omega \in \Omega$. By reflexivity, there exists a subsequence $\left\{\xi_{k}(\omega)\right\}$ of $\left\{\xi_{n}(\omega)\right\}$ such that $\xi_{k}(\omega)$ converges weakly to $\xi(\omega)$, where $\xi: \Omega \rightarrow X$ is a measurable mapping. Since $\xi_{n}(\omega)-\eta_{n}(\omega) \in(I-$ $T(\omega, \cdot))$, both $\xi_{n}(\omega)$ and $\xi_{n}(\omega)-\eta_{n}(\omega)$ converge to 0 . Since $I-T(\omega, \cdot)$ is a demiclosed mapping, $\xi(\omega) \in T(\omega, \xi(\omega))$.

Corollary 3.4: Let $X, T, W$ be as in Theorem 3.1 and suppose that there exists $\epsilon>0$ such that $L S(W, T(\omega, W), \epsilon ; X)$ is relatively compact for each $\omega \in \Omega$. If $I-T(\omega, \cdot)$ is demiclosed on $X$ for every $\omega \in \Omega$, then $T$ has a random fixed point.

Corollary 3.5: Let $X, T, W, L S(W, T(\omega, W) ; X)$ be as in Theorem 3.1. If the Banach space is reflexive and satisfies Opial's condition (that is, if $z_{n}$ converges weakly to $z$ and $z \neq v$ ), $\left.\liminf _{n \rightarrow \infty}\left\|z_{n}-z\right\|<\liminf _{n \rightarrow \infty}\left\|z_{n}-v\right\|\right)$, then $T$ has a random fixed point.

Corollary 3.6: Let $X, T, W, L S(W, T(\omega, W) ; X)$ be as in Theorem 3.1. If Banach space is uniformly bounded, then $T$ has a random fixed point. 
Theorem 3.7: Let $Y$ be a reflexive real Banach space which satisfies Opial's condition. Let $X$ be a separable closed convex subset of $Y$ and let $T: \Omega \times X \rightarrow \mathscr{K}(Y)$ be a Lipschitzian pseudocontractive random operator which satisfies the inwardness condition: for any $x \in X, T(\omega, x) \subseteq$ $\overline{I_{X}(x)}$, for each $\omega \in \Omega$. Suppose that there exist $x_{0} \in X$ and $\epsilon>0$ such that $\operatorname{LS}\left(x_{0}, \overline{C o}\left(T\left(\omega, x_{0}\right)\right)\right.$, $\epsilon ; X)$ is bounded for each $\omega \in \Omega$. If $T(\omega, X) \cap B_{r}$ is compact for any $B_{r}=\{z \in X:\|z\| \leq r\}$ then $T$ has a random fixed point.

Proof: Let $L$ be a Lipschitz measurable mapping of $T$. Select a measurable mapping $\alpha: \Omega \rightarrow(0, \infty)$ such that

$$
0<\alpha(\omega)<\min \left(L^{-1}(\omega), \epsilon\left(2 H\left(\left\{x_{0}\right\}, T\left(\omega, x_{0}\right)\right)+L(\omega) \epsilon\right)^{-1}\right) .
$$

Then, for any $y \in X$, define a contractive random operator $T_{y}: \Omega \times X \rightarrow \mathscr{K}(Y)$ by $T_{y}(\omega, x)=(1-$ $\alpha(\omega)) y+\alpha(\omega) T(\omega, x)$. The operator $T_{y}$ satisfies the inwardness condition: for any $x \in X$ $T_{y}(\omega, x) \subseteq \overline{I_{X}(x)}$ for each $\omega \in \Omega$.

Consider the mapping $G: \Omega \times X \rightarrow \mathscr{P}(X)$ defined by $G(\omega, y)=\left\{x: x \in T_{y}(\omega, x)\right\}$. For any $y \in X, G(\cdot, y): \Omega \rightarrow \mathscr{P}(X)$ is measurable [11, see proof of Theorem 3.1]. Also $G(\omega, y)$ is nonempty and closed for every $y \in X$ and $\omega \in \Omega$. It is easy to verify that

$$
G(\omega, y) \subseteq(1-\alpha(\omega)) y+\alpha(\omega) T(\omega, G(\omega, y))
$$

and

$$
\xi(\omega) \in G(\omega, \xi(\omega)) \text { iff } \xi(\omega) \in T(\omega, \xi(\omega))
$$

for each $\omega \in \Omega$ (where $\xi: \Omega \rightarrow X$ is a measurable map). For $u, v \in X$ (fixed),

$$
\|a-b\| \leq\|u-v\| \text { for any } a \in G(\omega, u) \text { and } b \in G(\omega, v) \text {, for each } \omega \in \Omega .
$$

Indeed $a \in G(\omega, u)$ and $b \in G(\omega, v)$ imply that

$$
\begin{gathered}
a=(1-\alpha(\omega)) u+\alpha(\omega) \eta, \quad \eta \in T(\omega, a), \\
b=(1-\alpha(\omega)) v+\alpha(\omega) \xi, \quad \xi \in T(\omega, b),
\end{gathered}
$$

and so, from pseudocontractivity of $T$, from (6) and (7) and choosing $r(\omega)<\alpha(\omega) /(1-\alpha(\omega))$ for each $\omega \in \Omega$,

$$
\begin{gathered}
\|a-b\| \leq\|(1+r(\omega))(a-b)-r(\omega)(\eta-\xi)\| \\
=\left\|(1+r(\omega))(a-b)-\frac{r(\omega)}{\alpha(\omega)}[a-(1-\alpha(\omega)) u-b+(1-\alpha(\omega)) v]\right\| \\
=\left\|(1+r(\omega))(a-b)-\frac{r(\omega)}{\alpha(\omega)}[(a-b)-(1-\alpha(\omega))(u-v)]\right\| \\
=\left\|\left(1+r(\omega)-\frac{r(\omega)}{\alpha(\omega)}\right)(a-b)-\frac{r(\omega)}{\alpha(\omega)}(1-\alpha(\omega))(u-v)\right\| \\
\leq\left(1+r(\omega)-\frac{r(\omega)}{\alpha(\omega)}\right)\|a-b\|+\frac{r(\omega)}{\alpha(\omega)}(1-\alpha(\omega))\|u-v\| .
\end{gathered}
$$

Therefore $r(\omega)(1-\alpha(\omega)) \alpha^{-1}(\omega)\|a-b\| \leq r(\omega)(1-\alpha(\omega)) \alpha^{-1}(\omega)\|u-v\|$, proving (5). 
It follows, in particular, that $G(\omega, y)$ belongs to $\mathfrak{C}^{B}(X)$ for any $y \in X$ and $\omega \in \Omega$. Besides, $T(\omega, G(\omega, y))$ is bounded (since $T$ is Lipschitzian), and by (3) we can conclude, under the hypothesis, for each $\omega \in \Omega, T(\omega, X) \cap B_{r}$ is compact for any $r$, that, for any $y \in X$, $G(\omega, y) \in \mathscr{G}(X)$. From

$$
H(G(\omega, x), G(\omega, y)) \leq \sup \{\|a-b\|: a \in G(\omega, x), b \in G(\omega, y)\}
$$

and from (5) it follows that the random operator $G: \Omega \times X \rightarrow \mathscr{K}(X)$ is nonexpansive, and by (4), has the same random fixed point of $T$. The set $L S\left(x_{0}, G\left(\omega, x_{0}\right) ; X\right)$ is bounded for each $\omega \in \Omega$ [16, proof of Theorem 8], Corollary 3.5 implies that $G$ has a random fixed point.

\section{Random Fixed Points of Firmly Nonexpansive Random Operators}

Let $C$ be a nonempty subset of a Banach space $X$, and let $\lambda \in(0,1)$. Then, a mapping $T: C \rightarrow X$ is said to be $\lambda$-firmly nonexpansive if

$$
\|T x-T y\| \leq\|(1-\lambda)(x-y)+\lambda(T x-T y)\|
$$

for all $x, y \in C$. In particular, if $(8)$ holds for every $\lambda \in(0,1)$, then $T$ is said to be a firmly nonexpansive mapping. It is clear that every $\lambda$-firmly nonexpansive mapping is nonexpansive. Conversely, with each nonexpansive mapping $T: C \rightarrow C$ one can associate a firmly nonexpansive mapping with the same fixed point set, whenever $C$ is closed and convex [6]. A random operator $T: \Omega \times C \rightarrow C$ is said to be $\lambda(\omega)$-firmly nonexpansive for some measurable mapping $\lambda: \Omega \rightarrow(0,1)$, if $T(\omega, \cdot)$ is $\lambda(\omega)$-firmly nonexpansive for each $\omega \in \Omega$. The aim of this section is to obtain a random fixed point theorem for a $\lambda(\omega)$-firmly nonexpansive random operator which is a stochastic analogue of the result by Smarzewski [19].

Theorem 4.1: Let $X$ be a separable uniformly convex Banach space, and let $C: \Omega \rightarrow \Im(X)$ be a measurable mapping defined by $C(\omega)=\bigcup_{k=1}^{n} C_{k}(\omega)$, where $C_{k}: \Omega \rightarrow \operatorname{CgC}(X)$ are measurable mappings for each $1 \leq k \leq n$. Suppose for any $\omega \in \Omega, T(\omega, \cdot): C(\omega) \rightarrow C(\omega)$ is $\lambda(\omega)$-firmly nonexpansive for some measurable map $\lambda: \Omega \rightarrow(0,1)$. Then $T$ has a random fixed point.

Proof: Let $\xi: \Omega \rightarrow X$ be a measurable mapping such that $\xi(\omega) \in C(\omega)$ for each $\omega \in \Omega$, and let $\left\{\xi_{k}(\omega)\right\}$ be the unique asymptotic center of the sequence $\left\{T^{i}(\omega, \xi(\omega))\right\}$ for each $\omega \in \Omega$ with respect to $C_{k}: \Omega \rightarrow \operatorname{CoBC}(X)(1 \leq k \leq n)$. In other words, by the uniform convexity of $X$, the measurable maps $\xi_{k}: \Omega \rightarrow X$, such that $\bar{\xi}_{k}(\omega) \in C_{k}(\omega)$ for each $\omega \in \Omega$, are uniquely determined by the identity

$$
f\left(\omega, \xi_{k}(\omega)\right)=\inf _{x \in C_{k}(\omega)} f(\omega, x),
$$

where for $x \in X$, the convex measurable map $f(\cdot, x): \Omega \rightarrow[0, \infty)$ is defined by $f(\omega, x)=\limsup _{i \rightarrow \infty}$ $\left\|x-T^{i}(\omega, \xi(\omega))\right\|$. Since $T$ is a nonexpansive random operator, we have

$$
\left\|T\left(\omega, \xi_{k}(\omega)\right)-T^{i+1}(\omega, \xi(\omega))\right\| \leq\left\|\xi_{k}(\omega)-T^{i}(\omega, \xi(\omega))\right\| .
$$

Hence,

$$
f\left(\omega, T\left(\omega, \xi_{k}(\omega)\right)\right) \leq f\left(\omega, \xi_{k}(\omega)\right)
$$

for all $k$ and $\omega \in \Omega$. Now, if $T\left(\omega, \xi_{k}(\omega)\right) \in C_{k}(\omega)$ for each $\omega \in \Omega$ and for some $k$, the uniqueness of an asymptotic center, in conjunction with (9) and (10), yields that $T\left(\omega, \xi_{k}(\omega)\right)=\xi_{k}(\omega)$ for 
each $\omega \in \Omega$. Otherwise, if $T\left(\omega, \xi_{k}(\omega) \notin C_{k}(\omega)\right.$ for all $k$ and $\omega \in \Omega$, then there exist integers $\left\{n_{1}\right.$, $\left.n_{2}, \ldots, n_{m}\right\} \subset\{1,2, \ldots, n\}, \quad(m \geq 2)$ such that $T\left(\omega, \xi_{n_{k}}(\omega)\right) \in C_{n_{k+1}}(\omega) \quad(k=1, \ldots, m-1)$ and $T\left(\omega, \xi_{n_{m}}(\omega)\right) \in C_{n_{1}}(\omega)$ for each $\omega \in \Omega$. Clearly, without loss of generality, one can rearrange the sequence of measurable mappings $C_{k}: \Omega \rightarrow \mathfrak{C B C}(X)$ in such a way that $n_{k}=k$ for all $k$. Hence one can combine (9) and (10) in order to get, for each $\omega \in \Omega$,

$$
\begin{gathered}
f\left(\omega, \xi_{1}(\omega)\right) \leq f\left(\omega, T\left(\omega, \xi_{m}(\omega)\right)\right) \leq f\left(\omega, \xi_{m}(\omega)\right) \\
\leq f\left(\omega, T\left(\omega, \xi_{m-1}(\omega)\right)\right) \leq f\left(\omega, \xi_{m-1}(\omega)\right) \ldots \\
\leq f\left(\omega, \xi_{2}(\omega)\right) \leq f\left(\omega, T\left(\omega, \xi_{1}(\omega)\right)\right) \leq f\left(\omega, \xi_{1}(\omega)\right) .
\end{gathered}
$$

Thus, we have

$$
f\left(\omega, T\left(\omega, \xi_{k}(\omega)\right)\right)=f\left(\omega, \xi_{k+1}(\omega)\right)
$$

for $\omega \in \Omega$. This, in view of the uniqueness of asymptotic centers $\xi_{k}: \Omega \rightarrow X$, yields that

$$
\xi_{k+1}(\omega)=T\left(\omega, \xi_{k}(\omega)\right) \text { for each } \omega \in \Omega \text { and } k=(1,2, \ldots, m)
$$

Here we denoted $\xi_{m+1}(\omega)=\xi_{1}(\omega)$ for the simplicity. Hence we readily derive for $\omega \in \Omega$,

$$
\begin{gathered}
\left\|\xi_{k-1}(\omega)-\xi_{k}(\omega)\right\|=\left\|T\left(\omega, \xi_{k}(\omega)\right)-T\left(\omega, \xi_{k+1}(\omega)\right)\right\| \\
\leq\left\|\xi_{k}(\omega)-\xi_{k+1}(\omega)\right\| \\
=\left\|T\left(\omega, \xi_{k-1}(\omega)\right)-T\left(\omega, \xi_{k}(\omega)\right)\right\| \\
\leq\left\|\xi_{k-1}(\omega)-\xi_{k}(\omega)\right\| \text { for every } k
\end{gathered}
$$

and

$$
\begin{gathered}
\left\|\xi_{1}(\omega)-\xi_{2}(\omega)\right\|=\left\|\xi_{2}(\omega)-\xi_{3}(\omega)\right\|=\ldots \ldots \ldots \\
\ldots \ldots \ldots=\left\|\xi_{m-1}(\omega)-\xi_{m}(\omega)\right\|=\left\|\xi_{1}(\omega)-\xi_{m}(\omega)\right\|=\gamma(\omega)
\end{gathered}
$$

where $\gamma: \Omega \rightarrow \mathbb{R}^{+}$is a measurable map.

Clearly, if $\gamma(\omega)=0$ for each $\omega \in \Omega$, then $\xi_{1}(\omega)=\xi_{2}(\omega)=T\left(\omega, \xi_{1}(\omega)\right)$ by (11). Thus it remains to show that the inequality $\gamma(\omega)>0$ for each $\omega \in \Omega$, is impossible. Since $T$ is $\lambda(\omega)$ firmly nonexpansive for some measurable map $\lambda: \Omega \rightarrow(0,1)$, it follows from (11) that

$$
\begin{gathered}
\gamma(\omega)=\left\|\xi_{k+1}(\omega)-\xi_{k}(\omega)\right\|=\left\|T\left(\omega, \xi_{k}(\omega)\right)-T\left(\omega, \xi_{k-1}(\omega)\right)\right\| \\
\leq\left\|(1-\lambda(\omega))\left(\xi_{k}(\omega)-\xi_{k-1}(\omega)\right)+\lambda(\omega)\left(\xi_{k+1}(\omega)-\xi_{k}(\omega)\right)\right\| \\
\leq(1-\lambda(\omega))\left\|\xi_{k}(\omega)-\xi_{k-1}(\omega)\right\|+\lambda(\omega)\left\|\xi_{k+1}(\omega)-\xi_{k}(\omega)\right\| \\
=\gamma(\omega) .
\end{gathered}
$$

Therefore, in view of the uniform convexity of the norm, we get 


$$
\begin{aligned}
& \xi_{k}(\omega)-\xi_{k-1}(\omega)=\alpha_{k}(\omega)\left(\xi_{k+1}(\omega)-\xi_{k}(\omega)\right) \\
& \left(2 \leq k \leq m, \xi_{m+1}(\omega)=\xi_{1}(\omega)\right) \text { for any } \omega \in \Omega
\end{aligned}
$$

for some non-zero real-valued measurable map $\alpha_{k}$. Moreover, by (12), we have $\left|\alpha_{k}(\omega)\right|=1$ for all $k$ and $\omega \in \Omega$. Hence, the following two cases may occur.

Case I: If $\alpha_{k}(\omega)=-1$ for each $\omega \in \Omega$ and for some $k$, then $\xi_{k-1}(\omega)=\xi_{k+1}(\omega)$. Therefore, one can apply the definition of a $\lambda(\omega)$-firmly nonexpansive operator and (11) to get, for each $\omega \in \Omega$,

$$
\begin{gathered}
\quad\left\|\xi_{k}(\omega)-\xi_{k-1}(\omega)\right\|=\left\|T\left(\omega, \xi_{k-1}(\omega)\right)-T\left(\omega, \xi_{k}(\omega)\right)\right\| \\
\leq\left\|(1-\lambda(\omega))\left(\xi_{k-1}(\omega)-\xi_{k}(\omega)\right)+\lambda(\omega)\left(\xi_{k}(\omega)-\xi_{k-1}(\omega)\right)\right\| \\
=\|1-2 \lambda(\omega)\|\left\|\xi_{k}(\omega)-\xi_{k-1}(\omega)\right\|<\left\|\xi_{k}(\omega)-\xi_{k-1}(\omega)\right\| .
\end{gathered}
$$

This contradiction shows that $\gamma(\omega)=0$, for each $\omega \in \Omega$.

Case II: If $\alpha_{k}(\omega)=1$ for each $\omega \in \Omega$ and for all $k$, we have

$$
\begin{gathered}
\xi_{2}(\omega)=\left(\xi_{1}(\omega)+\xi_{3}(\omega)\right) /_{2}, \xi_{3}(\omega)=\left(\xi_{2}(\omega)+\xi_{4}(\omega)\right) /{ }_{2} \ldots \ldots \\
\ldots \ldots \xi_{m-1}(\omega)=\left(\xi_{m-2}(\omega)+\xi_{m}(\omega)\right) /{ }_{2}, \xi_{m}(\omega)=\left(\xi_{1}(\omega)+\xi_{m-1}(\omega)\right) /{ }_{2} .
\end{gathered}
$$

Clearly, the first $m-2$ identities mean that the points $\xi_{2}(\omega), \xi_{3}(\omega), \ldots, \xi_{m-1}(\omega)$ divide the interval $\left[\xi_{1}(\omega), \xi_{m}(\omega)\right]$ into $m-1$ subintervals $\left[\xi_{k}(\omega), \xi_{k+1}(\omega)\right](1 \leq k \leq m-1)$ of same length $\gamma(\omega)>0$ for each $\omega \in \Omega$. But this leads to a contradiction with the identity $\xi_{m}(\omega)=\left(\xi_{1}(\omega)+\right.$ $\left.\xi_{m-1}(\omega)\right) / 2$ for each $\omega \in \Omega$. Hence we have always $\gamma(\omega)=0$ for $\omega \in \Omega$, which completes the proof.

\section{A Random Coincidence Point Theorem}

Jungck [12] gave the notion of compatible single valued mappings. Subsequently, Beg and Azam [1] introduced th notion of compatible multivalued mappings and proved various Banach type fixed point theorems for multivalued mappings. In this section we obtain a random coincidence point theorem and random fixed point theorem for random operators satisfying a contractive type condition.

Throughout this section, let $(X, d)$ be a Polish space, that is, a separable complete metric space. Mappings $T: X \rightarrow \mathfrak{C B}(X)$ and $f: X \rightarrow X$ are compatible if, whenever there is a sequence $\left\{x_{n}\right\}$ in $X$ satisfying $\lim _{n \rightarrow \infty} f x_{n} \in \lim _{n \rightarrow \infty} T x_{n}$ (provided $\lim _{n \rightarrow \infty} f x_{n}$ exists in $X$ and $\lim _{n \rightarrow \infty} T x_{n}$ exists in $\mathcal{C B}(X)$ ), $\lim _{n \rightarrow \infty} H\left(f T x_{n}, T f x_{n}\right)=0$. For details we refer to Beg and Azam [1]. Random operators $f: \Omega \times$ $X \rightarrow X$ and $T: \Omega \times X \rightarrow \mathcal{C} \mathfrak{B}(X)$ are compatible if $f(\omega, \cdot)$ and $T(\omega, \cdot)$ are compatible for each $\omega \in \Omega$. (See Beg and Shahzad [2].)

Theorem 5.1: Let $T: \Omega \times X \rightarrow \mathfrak{C B B}(X)$ be a random multivalued operator and let $f: \Omega \times X \rightarrow X$ be a continuous random operator, such that $T(\omega, X) \subseteq f(\omega, X)$ for every $\omega \in \Omega$. If $f$ and $T$ are compatible and for all $x, y \in X$ and $\omega \in \Omega$,

$$
H(T(\omega, x), T(\omega, y)) \leq \lambda(\omega) d(f(\omega, x), f(\omega, y))
$$


(where $\lambda: \Omega \rightarrow(0,1)$ is a measurable map), then there is a random coincidence point of $f$ and $T$.

Proof: Let $\xi_{0}: \Omega \rightarrow X$ be an arbitrary measurable mapping. Let $\xi_{1}: \Omega \rightarrow X$ be a measurable mapping such that $y_{1}: \Omega \rightarrow X$ defined by $y_{1}(\omega)=f\left(\omega, \xi_{1}(\omega)\right) \in T\left(\omega, \xi_{0}(\omega)\right)$. The existence of a measurable map $y_{1}: \Omega \rightarrow X$ is due to Kuratowski and Ryll-Nardzewski [14]. Using Itoh [10, Proposition 4] and the fact that $T(\omega, X) \subseteq f(\omega, X)$ for every $\omega \in \Omega$, we may choose a measurable mapping $\xi_{2}: \Omega \rightarrow X$ such that for each $\omega \in \Omega$, it holds true that

$$
y_{2}(\omega)=f\left(\omega, \xi_{2}(\omega)\right) \in T\left(\omega, \xi_{1}(\omega)\right)=A_{1}(\omega)
$$

and

$$
\begin{gathered}
d\left(y_{1}(\omega), y_{2}(\omega)\right)=d\left(f\left(\omega, \xi_{1}(\omega)\right), f\left(\omega, \xi_{2}(\omega)\right)\right) \\
\leq H\left(T\left(\omega, \xi_{0}(\omega)\right), T\left(\omega, \xi_{1}(\omega)\right)\right)+\lambda(\omega) .
\end{gathered}
$$

Since for each $\omega \in \Omega, T(\omega, X) \subseteq f(\omega, X)$ by [10, Proposition 4], there exists a measurable selector $f\left(\cdot, \xi_{3}(\cdot)\right)=y_{3}: \Omega \rightarrow X$ of $T\left(\cdot, \xi_{2}(\cdot)\right)$ such that for any $\omega \in \Omega$,

$$
\begin{gathered}
d\left(y_{2}(\omega), y_{3}(\omega)\right)=d\left(f\left(\omega, \xi_{2}(\omega)\right), f\left(\omega, \xi_{3}(\omega)\right)\right) \\
\leq H\left(T\left(\omega, \xi_{1}(\omega)\right), T\left(\omega, \xi_{2}(\omega)\right)\right)+\lambda^{2}(\omega) .
\end{gathered}
$$

By induction, we form sequences of measurable mappings such that for each $\omega \in \Omega$ and $n>0$,

$$
y_{n}(\omega)=f\left(\omega, \xi_{n}(\omega)\right) \in T\left(\omega, \xi_{n-1}(\omega)\right)=A_{n-1}(\omega) .
$$

Furthermore, for any $\omega \in \Omega$,

$$
\begin{gathered}
d\left(y_{n+1}(\omega), y_{n+2}(\omega)\right)=d\left(f\left(\omega, \xi_{n+1}(\omega)\right), f\left(\omega, \xi_{n+2}(\omega)\right)\right) \\
\leq H\left(T\left(\omega, \xi_{n}(\omega)\right), T\left(\omega, \xi_{n+1}(\omega)\right)\right)+\lambda^{n+1}(\omega)
\end{gathered}
$$

and

$$
\begin{gathered}
d\left(y_{n+1}(\omega), y_{n+3}(\omega)\right)=d\left(f\left(\omega, \xi_{n+2}(\omega)\right), f\left(\omega, \xi_{n+3}(\omega)\right)\right) \\
\leq H\left(T\left(\omega, \xi_{n+1}(\omega)\right), T\left(\omega, \xi_{n+2}(\omega)\right)\right)+\lambda^{n+2}(\omega) .
\end{gathered}
$$

Hence,

$$
\begin{gathered}
d\left(f\left(\omega, \xi_{n+1}(\omega)\right), f\left(\omega, \xi_{n+2}(\omega)\right)\right) \leq \lambda(\omega) d\left(f\left(\omega, \xi_{n}(\omega)\right), f\left(\omega, \xi_{n+1}(\omega)\right)\right)+\lambda^{n+1}(\omega) \\
\leq \lambda(\omega)\left\{H\left(T\left(\omega, \xi_{n-1}(\omega)\right), T\left(\omega, \xi_{n}(\omega)\right)\right)+\lambda^{n}(\omega)\right\}+\lambda^{n+1}(\omega) \\
\leq \lambda^{2}(\omega) d\left(f\left(\omega, \xi_{n-1}(\omega)\right), f\left(\omega, \xi_{n}(\omega)\right)\right)+2 \lambda^{n+1}(\omega) \\
\ldots \ldots \ldots \ldots \ldots \ldots \ldots \ldots \ldots \ldots \ldots \\
\ldots \ldots \ldots \ldots \ldots \ldots \ldots \ldots
\end{gathered}
$$


Similarly,

$$
\begin{gathered}
d\left(f\left(\omega, \xi_{n+2}(\omega)\right), f\left(\omega, \xi_{n+3}(\omega)\right)\right) \leq \lambda^{n+1}(\omega) d\left(f\left(\omega, \xi_{1}(\omega)\right)\right. \\
\left.f\left(\omega, \xi_{2}(\omega)\right)\right)+(n+1) \lambda^{n+2}(\omega) .
\end{gathered}
$$

Hence, $\left\{y_{n}(\omega)\right\}$ is a Cauchy sequence. Indeed, let $\omega \in \Omega$ be an arbitrary fixed point, let $m<n$. Then,

$$
\begin{gathered}
d\left(y_{m+1}(\omega), y_{n}(\omega)\right)=d\left(f\left(\omega, \xi_{m+1}(\omega)\right), f\left(\omega, \xi_{n}(\omega)\right)\right) \\
\leq d\left(f\left(\omega, \xi_{m+1}(\omega)\right), f\left(\omega, \xi_{m+2}(\omega)\right)\right) \\
+d\left(f\left(\omega, \xi_{m+2}(\omega)\right), f\left(\omega, \xi_{m+3}(\omega)\right)\right)+\ldots \\
\ldots \ldots+d\left(f\left(\omega, \xi_{n-1}(\omega)\right), f\left(\omega, \xi_{n}(\omega)\right)\right)
\end{gathered}
$$

Now, (14) and (15) imply that

$$
\begin{gathered}
d\left(y_{m+1}(\omega), y_{n}(\omega)\right) \leq\left\{\lambda^{m}(\omega) d\left(f\left(\omega, \xi_{1}(\omega)\right), f\left(\omega, \xi_{2}(\omega)\right)\right)+m \lambda^{m+1}(\omega)\right\} \\
+\left\{\lambda^{m+1}(\omega) d\left(f\left(\omega, \xi_{1}(\omega)\right), f\left(\omega, \xi_{2}(\omega)\right)\right)+(m+1) \lambda^{m+2}(\omega)\right\} \\
+\ldots \ldots \ldots \ldots \ldots \ldots \ldots \ldots \ldots \ldots \\
+\left\{\lambda^{n-2}(\omega) d\left(f\left(\omega, \xi_{1}(\omega)\right), f\left(\omega, \xi_{2}(\omega)\right)\right)+(n-2) \lambda^{n-1}(\omega)\right\}
\end{gathered}
$$

It further implies that

$$
d\left(y_{m+1}(\omega), y_{n}(\omega)\right) \leq \sum_{i=m}^{n-2}\left[\lambda^{i}(\omega) d\left(f\left(\omega, \xi_{1}(\omega)\right), f\left(\omega, \xi_{2}(\omega)\right)\right)+i \lambda^{i+1}(\omega)\right]
$$

Thus, $\left\{y_{n}(\omega)\right\}$ is a Cauchy sequence. By completeness of the space, there exists $\gamma(\omega) \in X$, such that for each $\omega \in \Omega, d\left(y_{n}(\omega), \gamma(\omega)\right) \rightarrow 0$ as $n \rightarrow \infty$. (The mapping $\gamma: \Omega \rightarrow X$ is a pointwise limit of the measurable mappings $\left\{y_{n}\right\}$, therefore it is measurable.) The continuity of $f$ implies that

$$
d\left(f\left(\omega, y_{n}(\omega)\right), f(\omega, \gamma(\omega))\right) \rightarrow 0
$$

It further implies that

$$
\begin{gathered}
H\left(T\left(\omega, y_{n}(\omega)\right), T(\omega, \gamma(\omega))\right) \leq \lambda(\omega) d\left(f\left(\omega, y_{n}(\omega)\right), f(\omega, \gamma(\omega))\right) \\
<d\left(f\left(\omega, y_{n}(\omega)\right), f(\omega, \gamma(\omega))\right) \rightarrow 0 .
\end{gathered}
$$

Inequality (13) and the fact that $\left\{f\left(\omega, \xi_{n}(\omega)\right)\right\}$ is a Cauchy sequence imply that there exists $A(\omega) \in \mathcal{C} \mathscr{B}(X)$ such that $T\left(\omega, \xi_{n}(\omega)\right) \rightarrow A(\omega)$. (By Itoh [10, Proposition 1], $A$ is measurable.) Furthermore, for each $\omega \in \Omega$,

$$
d(\gamma(\omega), A(\omega))=\lim _{n \rightarrow \infty} d\left(y_{n}(\omega), A_{n}(\omega)\right) \leq \lim _{n \rightarrow \infty} H\left(A_{n-1}(\omega), A_{n}(\omega)\right)=0 .
$$

It further implies that 


$$
\lim _{n \rightarrow \infty} f\left(\omega, \xi_{n}(\omega)\right)=\gamma(\omega) \in A(\omega)=\lim _{n \rightarrow \infty} T\left(\omega, \xi_{n}(\omega)\right) .
$$

Hence by compatibility of $f$ and $T$, we have

$$
\lim _{n \rightarrow \infty} H\left(f\left(\omega, T\left(\omega, \xi_{n}(\omega)\right)\right), T\left(\omega, f\left(\omega, \xi_{n}(\omega)\right)\right)\right)=0,
$$

for each $\omega \in \Omega$. Therefore

$$
\lim _{n \rightarrow \infty} d\left(f\left(\omega, y_{n+1}(\omega)\right), T\left(\omega, y_{n}(\omega)\right)\right)=0 .
$$

Hence, $d(f(\omega, \gamma(\omega)), T(\omega, \gamma(\omega)))=0$ for every $\omega \in \Omega$. That is, $f(\omega, \gamma(\omega)) \in T(\omega, \gamma(\omega))$, for each $\omega \in \Omega$.

Corollary 5.2: If in addition to the hypothesis of Theorem 5.1, the following condition is satisfied: for each $\omega \in \Omega$,

$$
\begin{gathered}
f(\omega, \gamma(\omega)) \in T(\omega, \gamma(\omega)) \text { implies } \\
\lim _{n \rightarrow \infty} f(\omega, \gamma(\omega))^{n}=t(\omega),
\end{gathered}
$$

then $t$ is a common random fixed point of $f$ and $T$.

Proof: By Theorem 5.1, there exists a measurable map $\gamma: \Omega \rightarrow X$ such that $f(\omega, \gamma(\omega)) \in$ $T(\omega, \gamma(\omega))$ for $\omega \in \Omega$. Obviously,

$$
f\left(\omega \lim _{n \rightarrow \infty} f(\omega, \gamma(\omega))^{n}\right)=f(\omega, t(\omega)) .
$$

Thus, $t(\omega)=f(\omega, t(\omega))$ for each $\omega \in \Omega$.

Therefore, by [2, Remarks 3.3], we have

$$
f(\omega, \gamma(\omega))^{n+1} \in T\left(\omega, f(\omega, \gamma(\omega))^{n}\right)
$$

for any $\omega \in \Omega$. It further implies that $t(\omega) \in T(\omega, t(\omega))$ for each $\omega \in \Omega$.

\section{Acknowledgements}

The authors are grateful to Professor Jewgeni H. Dshalalow and the referee for helpful suggestions which improved the presentation of the paper.

\section{References}

[1] Beg, I. and Azam, A., Fixed points of asymptotically regular multivalued mappings, J. Austral. Math. Soc. (Ser. A) 53 (1992), 313-326.

[2] Beg, I. and Shahzad, N., Random fixed points of random multivalued operators on Polish spaces, Nonlinear Anal. 20:7 (1993), 835-847.

[3] Bharucha-Reid, A.T., Fixed point theorems in probabilistic analysis, Bull. Amer. Math. Soc. 82 (1976), 641-657.

[4] Fan, K., Extensions of two fixed point theorems for F.E. Browder, Math. Z. 112 (1969), 234-240. 
[5] Goebel, K. and Kuczumov, T., A contribution to the theory of nonexpansive mappings, Bull. Calcutta Math. Soc. 70 (1978), 355-357.

[6] Goebel, K. and Reich, S., Uniform Convexity, Hyperbolic Geometry, and Nonexpansive Mappings, Marcel Dekker, New York 1984.

[7] Hans, O., Reduzierende zulällige Transformaten, Czechoslovak Math. J. 7 (1957), 154-158.

[8] Hans, O., Random operators equations, In: Proc. 4th Berkeley Symposium on Mathematical Statistics and Probability, Vol. II, Part I, 185-202, University of California Press, Berkeley 1961.

[9] Himmelberg, C.J., Measurable relations, Fund. Math. 87 (1975), 53-72.

[10] Itoh, S., A random fixed point theorem for a multivalued contraction mapping, Pacific J. Math. 68 (1977), 85-90.

[11] Itoh, S., Ransom fixed point theorems with an application to random differential equations in Banach spaces, J. Math. Anal. Appl. 67 (1979), 261-273.

[12] Jungck, G., Common fixed points for commuting and compatible maps on compacta, Proc. Amer. Math. Soc. 103:3 (1988), 977-983.

[13] Kirk, W.A. and Ray, W.O., Fixed point theorems for mappings defined on unbounded sets in Banach spaces, Studia Math. 64 (1979), 127-138.

[14] Kuratowski, K. and Ryll-Nardzewski, C., A general theorem on selectors, Bull. Acad. Polon. Sci. Ser. Sci. Math. Astronom. Phys. 13 (1965), 397-403.

[15] Lin, T.C., Random approximations and random fixed point theorems for nonself maps, Proc. Amer. Math. Soc. 103 (1988), 1129-1135.

[16] Marino, G., Fixed points for multivalued mappings defined on unbounded sets in Banach spaces, J. Math. Anal. Appl. 157 (1991), 555-566.

[17] Papageorgiou, N.S., Random fixed point theorems for measurable multifunctions in Banach spaces, Proc. Amer. Math. Soc. 97 (1986), 507-514.

[18] Sehgal, V.M. and Singh, S.P., On random approximations and a random fixed point theorem for set-valued mappings, Proc. Amer. Math. Soc. 95 (1985), 91-94.

[19] Smarzewski, R., On firmly nonexpansive mappings, Proc. Amer. Math. Soc. 113 (1991), 723-725.

[20] Spacek, A., Zufällige Gleichungen, Czechoslovak Math. J. 5 (1955), 462-466. 


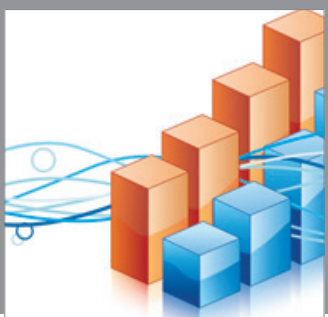

Advances in

Operations Research

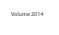

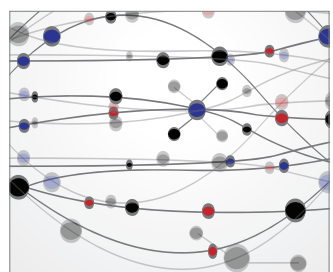

\section{The Scientific} World Journal
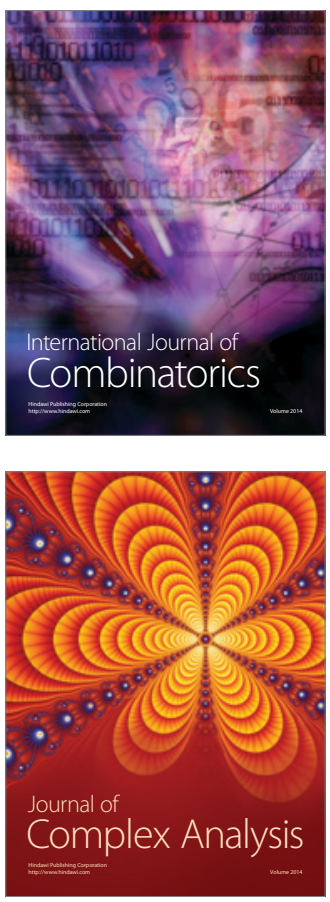

International Journal of

Mathematics and

Mathematical

Sciences
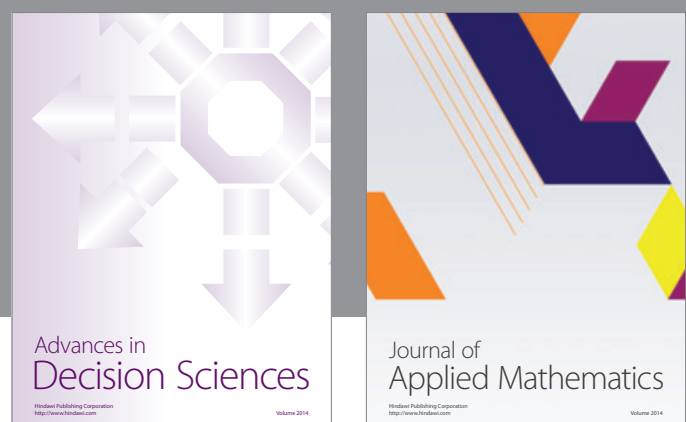

Journal of

Applied Mathematics
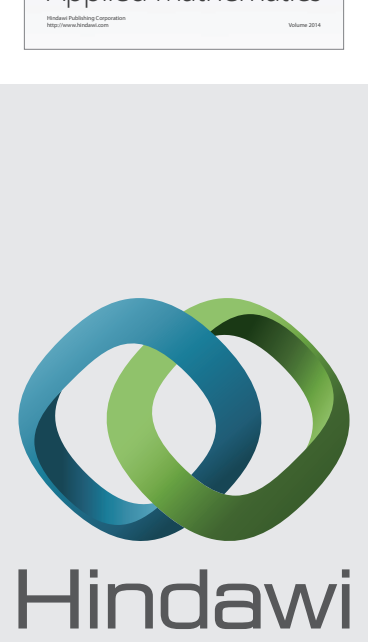

Submit your manuscripts at http://www.hindawi.com
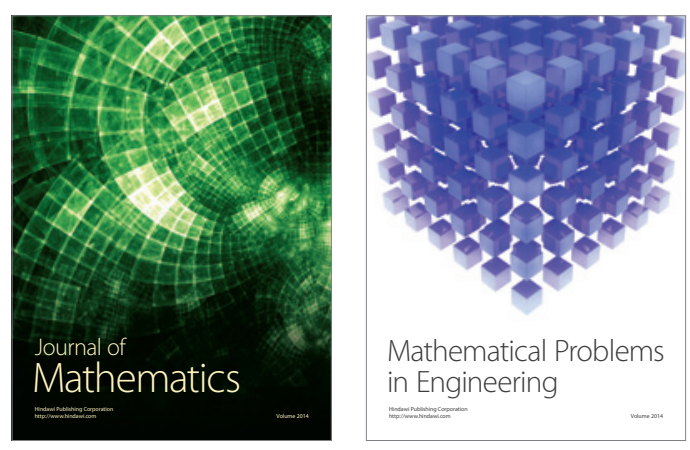

Mathematical Problems in Engineering
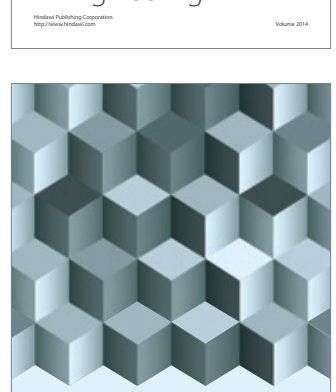

Journal of

Function Spaces
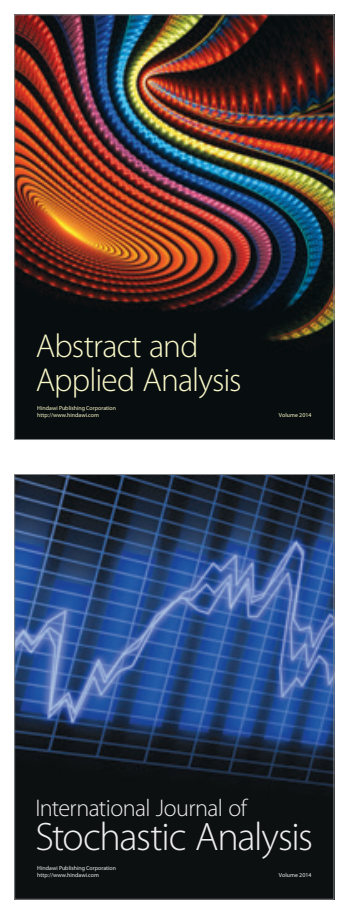

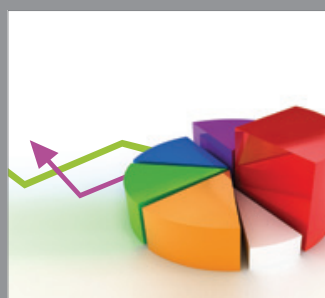

ournal of

Probability and Statistics

Promensencen
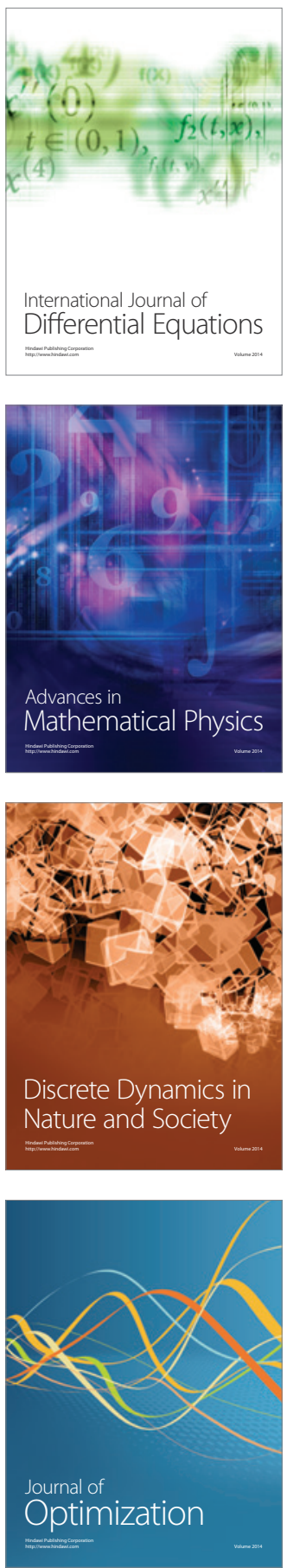